\title{
Evaluation of Osseous Changes in Oral Carcinoma - 2D Vs 3D Imaging
}

\section{IJCRR}

Section: Healthcare

Sci. Journal Impact

Factor: 6.1 (2018)

ICV: 90.90 (2018)

(c) (i) (8)

Copyright@IJCRR

\section{Abhinaya LM ${ }^{1}$, Muthukrishnan Arvind ${ }^{2}$, Deepika Rajendran ${ }^{3}$}

'Department of Oral Medicine and Radiology and Special Care Dentistry, Saveetha Dental College and Hospitals, Saveetha Institute of Medical and Technical Sciences (SIMATS), Saveetha University, Chennai-77, India; ${ }^{2}$ Professor and Head, Department of Oral Medicine and Radiology and Special Care Dentistry, Saveetha Dental College and Hospitals, Saveetha Institute of Medical and Technical Sciences (SIMATS), Saveetha University, Chennai-77, India; ${ }^{3}$ Senior Lecturer, Department of Oral Medicine and Radiology and Special Care Dentistry, Saveetha Dental College and Hospitals, Saveetha Institute of Medical and Technical Sciences (SIMATS), Saveetha University, Chennai-77, India.

\section{ABSTRACT}

Introduction: Oral cancer is the eighth most common cancer and accounts for $2 \%$ of cancer death worldwide. In India, Oral cancer accounts for $30-50 \%$ of all cancers; with squamous cell carcinoma the most common. Radiologic assessment of bone invasion plays a major role to evaluate the extent of surgical bone resection.

Objective: This study compares osseous changes in OSCC using Orthopantomogram (OPG) and Cone Beam Computed Tomography (CBCT).

Methods: A retrospective analysis of patients who visited the Department of Radiology from June 2019 to March 2020 was included in the study. After reviewing 67 case records, 12 patients who were clinically and histopathologically proven as OSCC patients, and had undergone imaging, both OPG and CBCT for assessment were selected.

Results: The study subjects included 4 female (33.3\%) patients and 8 male $(66.6 \%)$ patients. The predominant age group in the study was $40-50$ years $(41.7 \%)$. OSCC frequently involved mandible $(75 \%)$; the posterior region $(91.7 \%)$ and the commonly reported TNM staging were stage IV-A (66.6\%) followed by stage-III (33.3\%). Comparison of the OSCC and internal structure appearance in OPG there was a positive correlation seen and in comparison, to CBCT it was not statistically significant. On comparison for the involvement of adjacent structures and OSCC in OPG and CBCT, there was no significant difference noticed.

Conclusion: CBCT can be recommended for imaging oral carcinoma of the maxillofacial region replacing $2 \mathrm{D}$ and high exposure medical CT imaging.

Key Words: Cancer, CBCT, Imaging, OSCC, OPG, Squamous cell carcinoma

\section{INTRODUCTION}

Oral cancer is eighth most common cancers and an estimated amount of about 378,500 new cases of intraoral cancer are diagnosed worldwide. ${ }^{1}$ The global incidence of lip, oral cavity, and pharyngeal cancer are 529,500 equate to $3.8 \%$ of all carcinoma cases and is predicted to rise by $62 \%$ to 856,000 cases by $2035 .^{2}$

Early tissue changes that happen due to various oral habits such as smoking and chewing tobacco ${ }^{3}$ or stress are termed as Oral potentially malignant disorders (OPMDs). ${ }^{4,5} \mathrm{~A}$ recent report suggests that $16 \%-62 \%$ of the potentially malignant disorders undergo malignant transformation. ${ }^{6}$ Oral cancer represents more than $50 \%$ of all cancers; most common cancer among male, third most common among female and is commonly related to deleterious habits such as tobacco chewing, betel quid chewing, tobacco smoking, reverse smoking, alcohol consumption along with low socio-economic status, poor diet and hygiene, viral infections, chronic irritation from dentures or fractured teeth. ${ }^{1}$ They have proven to have an effect on overall as well as oral health of the patients. ${ }^{7,8}$ The evaluation of carcinogenic risks of smokeless tobacco by the International Agency on Research for Cancer (IARC) has confirmed that smoking tobacco is carcinogenic to humans and the main target organ being the oral cavity where the products are applied locally. ${ }^{9}$ However, several studies in recent times have reported increasing incidence of cancer in patients with no significant tobacco and alcohol exposure. ${ }^{10,11}$ OSCC is the most prevalent oral malignancy ${ }^{12}$ and is often reported with rapid growth occurring even with-

\section{Corresponding Author:}

Muthukrishnan Arvind, Professor and Head, Department of Oral Medicine and Radiology and Special Care Dentistry, Saveetha Dental College and Hospitals, Saveetha Institute of Medical and Technical Sciences (SIMATS), Saveetha University, Chennai-77, India; Phone: 9444303303; Email: arvindm@saveetha.com

ISSN: 2231-2196 (Print)

Received: 16.09 .2020
ISSN: 0975-5241 (Online)

Revised: 17.10 .2020
Accepted: 14.11 .2020
Published: 26.12 .2020 
out previous clinical signs, hence clinical examination must be completed with radiographic examination for the assessment of size, thickness, depth, and bone invasion. This bone invasion, cortical or medullary is an indication of the T4 tumor stage and at this level of extension, the survival rate is close to $50 \%$ whether the treatment is surgical management or chemotherapy. ${ }^{13}$ For this very reason, the detection of bone invasion significantly improves patient prognosis. ${ }^{14}$ Studies have shown that vitamin $C$ exhibits analgesic properties and is proven to have a potential role in the improvement of overall quality of life in cancer patients. ${ }^{15}$ Complementing the clinical and histopathological diagnosis ${ }^{16}$, radiographic imaging plays a vital role. Owing to easy availability and lower radiation dose, the commonly used imaging method is orthopantomogram and it was found to have $92 \%$ sensitivity for the detection of mandibular involvement by malignancies followed by CBCT, CT, and MRI. ${ }^{17,18} \mathrm{CBCT}$ imaging ${ }^{19}$ has also shown promise, as the use of three-dimensional information in dentomaxillofacial radiology has consistently grown with recent units, allowing the recovery of tissue sections with a thickness of up to $0.1 \mathrm{~mm} \cdot{ }^{20}$ Few other common indications for CBCT in dentistry include assessment of the jaws for placement of implants; examination of teeth and facial structures for orthodontic treatment planning; evaluation of TMJ for osseous degenerative changes, evaluation of mandibular third molar ${ }^{21}$; endodontic cases such as for root resorption ${ }^{22}$; evaluation of teeth and bone for cysts and tumors. According to the literature, the occurrence of osteonecrosis of the jaw after tooth extraction among patients with cancer ranges from $1.6 \%$ to $14.8 \% .^{23}$

Preoperative evaluation of CBCT imaging provides the reliability of anatomical measurements of bone invasion and surgical planning. ${ }^{24}$ One other major advantage of $\mathrm{CBCT}$ and OPG, being the patient's soft-tissue images are not distorted due to gravity.

The aim of our study was a comparison of osseous changes detected in OSCC using OPG and CBCT imaging modalities.

\section{MATERIALS AND METHOD}

A retrospective study was conducted in the Department of Radiology from June 2019 to March 2020. After reviewing 67 case records, 12 cases who were clinically and histopathologically proven OSCC were reviewed, and the radiographic images, both OPG and CBCT were obtained. The study was approved by the scientific review board (SRB) and institutional ethical committee (Approval number SDC/ SIHEC/2020/DIASDATA /0619-0320). Two researchers were involved in the study, a primary researcher and a department faculty. Cross-verification was done using records and clinical photographs. Sampling bias was minimized as there was no sorting process involved and all the data was included.

We included patients who had undergone both OPG and CBCT imaging prior to any surgical intervention and were graded and diagnosed as OSCC. We excluded other carcinoma diagnosis and patients with prior CT, MRI reports.

Data Collection was done from the previous records in the department. The retrieved data was formulated in an excel sheet and then imported into SPSS (IBM20). The dependent variables included osseous changes seen in OPG and CBCT and involvement of surrounding structures. The independent variables included were age, sex, site, and TNM staging.

\section{Statistical Analysis}

Descriptive analysis was performed using Chi-square test (IBM SPSS 20) and the analysis used was correlation and association.

\section{RESULTS AND DISCUSSION}

In this retrospective study of 12 patients who were diagnosed with OSCC; the patients OPG and CBCT were analyzed. Out of 12 patients, 8 patients were male $(66.6 \%)$ and 4 patients were female (33.3\%) [Graph 1].

Frequency distribution of age of patient was higher in 40 - 50 years $(41.7 \%)$ followed by $50-60$ years $(33.3 \%)$ and $60-70$ years (16.7\%); 20-30 years (8.3\%) [Graph 2]. Frequency distribution of site involved maximum reported in the mandible (75\%) when compared to that of the maxilla $(25 \%)$ and the most affected region was the posterior $(91.7 \%)$ than the anterior region (8.3\%) [Graph 3].

Frequency distribution of TNM staging involving Stage IV-A $(66.6 \%)$ is commonly diagnosed followed by Stage-III (33.3\%) [Graph 4]. Descriptive analysis to see the association between OSCC and internal structure in OPG and CBCT using the Chi-square test which was statistically significant [Graph 5,6]. Descriptive analysis to see the association between OSCC and involvement of adjacent structures in OPG and $\mathrm{CBCT}$ using the Chi-square test [Graph 7,8].

OSCC accounts for approximately $90 \%$ of malignant oral lesions and is widely the most commonly occurring malignant tumor of oral tissues and the mortality rate of OSCC is relatively high, with a five-year survival rate of $50 \%{ }^{25}$ In the present study, an increased incidence of OSCC was found in males than in females which were consistent with the studies done by Tandon et al ${ }^{1}$ and Singh MP et al. ${ }^{26} 95 \%$ of oral cancers have occurred in individuals over 40 years of age which is similar to our study where the patient fell under $40-50$ years $(41.7 \%)$ was seen. The mean age of presentation of oral cancer is the fifth and sixth decade of life in Asian 
populations. ${ }^{27}$ This result was in concordance to the study done by Tandon et $\mathrm{al}^{1}$ and $\mathrm{Rao}$ et $\mathrm{al}^{28}$ whose mean patient age was 53.4 years.

Similar to available literature, the mandible was the most commonly involved Jaw $(75 \%)$ in this study followed by maxilla $(25 \%)$ which is seen in a study done by Shah et al. ${ }^{29}$ The region affected is contradicting to our study where the posterior region was commonly affected ramus and body of mandible and maxilla posterior alveolar bone. The TNM staging of our study group was Stage-IV (66.6\%) which was similar to the study done by Czerwonka et al. ${ }^{30}$ followed by Stage III (33.3\%). In the comparison of extension of the lesion between OPG and CBCT; OPG revealed extension up to the inferior border of mandible and extension towards the ascending ramus of the mandible. A study done by Hakim et al. ${ }^{31}$ states that there was a higher sensitivity to cortical bone expansion seen in CBCT when compared to other imaging modalities which were similar to our study wherein there was a better appreciation of the expansion of cortical plate and obliteration of the maxillary sinus floor when compared to OPG. Involvement of IAN canal was concordance to our study was done by Bhaumik and Joshi et $\mathrm{al}^{32}$ on seeing the comparison between the radiographic feature in $\mathrm{OPG}$ and $\mathrm{CBCT}$; OPG revealed floating tooth appearance due to the rapidly growing malignant lesion and revealed tooth displacement, saucerization of bone and in CBCT with cortical bone expansion and obliteration into the floor of the maxillary sinus which was similar to the study done by Shah PH et al . ${ }^{29}$

\section{CONCLUSION}

To conclude, the radiographic investigation is mandatory to assess bone invasion in OSCC involving the jaws. With the added advantage of imaging in three dimensions, CBCT should be the sole imaging modality to assess bone involvement. 2-D imaging need not be undertaken as an investigative aid. Since 3-D imaging is freely available and more diagnostic in routine practice, for intraosseous lesions 3-D imaging can be the sole diagnostic imaging.

\section{ACKNOWLEDGEMENTS}

The authors thank the Saveetha Dental College for giving permission to access the electronic database for data collection for our study.

\section{Authors Contribution}

Abhinaya LM has made substantial contributions towards study design, acquiring an analysis of data, drafting the final paper, and revising it critically.
M.Arvind has made substantial contributions towards study design, acquiring an analysis of data, drafting the final paper, and revising it critically.

Deepika Rajendran has made substantial contributions in proofreading and final drafting.

\section{Conflict of Interest}

NIL

\section{Source of Funding}

Self

\section{REFERENCES}

1. Tandon A, Bordoloi B, Jaiswal R et al. Demographic and clinicopathological profile of oral squamous cell carcinoma patients of North India: A retrospective institutional study. SRM J Res Dent Sci 2018;9:114.

2. Petti S, Masood M, Scully C. The Magnitude of Tobacco Smoking-Betel Quid Chewing-Alcohol Drinking Interaction Effect on Oral Cancer in South-East Asia. A Meta-Analysis of Observational Studies. PLoS ONE 2013;8:e78999.

3. Shelgikar P, Subodhini A, Iyer C. Evaluation of Nitric oxide levels and arginase activity in oral cancer patients. Int $\mathrm{J}$ Curr Res Rev 2016;8(11):34-37.

4. Venugopal A, Maheswari TNU. Expression of matrix metalloproteinase-9 in oral potentially malignant disorders: a systematic review. J Oral Maxillofac Pathol 2016; 20(3): 474-479.

5. Muthukrishnan A, Kumar LB. Actinic cheilosis: early intervention prevents malignant transformation. Case Rep 2017;2017:bcr2016218654.

6. Maheswari TNU, Venugopal A, Sureshbabu N, et al. Salivary micro RNA as a potential biomarker in oral potentially malignant disorders: A systematic review. Tzu Chi Med J 2018;30.

7. Subashri A, Uma Maheshwari TN. Knowledge and Attitude of Oral Hygiene Practice among Dental Students. Res J Pharm Tech 2016;9:1840-1842.

8. Dharman S, Muthukrishnan A. Oral mucous membrane pemphigoid - Two case reports with varied clinical presentation. J Ind Soc Periodontol 2016;20:630.

9. Warnakulasuriya S, Muthukrishnan A. Oral health consequences of smokeless tobacco use. Ind J Med Res 2018;148:35.

10. Toporcov TN, Znaor A, Zhang ZF, et al. Risk factors for head and neck cancer in young adults: a pooled analysis in the INHANCE consortium. Int J Epidemiol 2015;44:169-185.

11. Ranganathan K, Rooban T, Rao UM. Oral squamous cell carcinoma in patients with and without predisposing habits in glossal and extra-glossal site: An institutional experience in South India. Indian J Cancer 2015;52:625.

12. Misra SR, Shankar Y, Rastogi V, Maragathavalli G. Metastatic hepatocellular carcinoma in the maxilla and mandible, an extremely rare presentation. Contemp Clin Dent 2015 Mar;6(Suppl 1):S117-21

13. Chaitanya N, Muthukrishnan A, Gandhi Babu D, Kumari CS, Lakshmi MA, Palat G, et al. Role of vitamin E and vitamin A in oral mucositis induced by cancer chemo/radiotherapy-a metaanalysis. J Clin Diagn Res. 2017 May; 11(5): ZE06-ZE09.

14. Uribe S, Rojas LA, Rosas CF. Accuracy of imaging methods for detection of bone tissue invasion in patients with oral squamous cell carcinoma. Dentomaxillofacial Radiol 2013;42(6):20120346. 
15. Chaitanya N, Muthukrishnan A, Krishnaprasad CMS, Sanjuprasanna G, Pillay P, Mounika B. An insight and update on the analgesic properties of vitamin C. J Pharm Bioallied Sci. 2018 Jul-Sep; 10(3): 119-125.

16. Steele JC, Clark HJ, Hong CHL, et al. World workshop on oral medicine VI: an international validation study of clinical competencies for advanced training in oral medicine. Oral Surg Oral Med Oral Pathol Oral Radiol 2015 Aug;120(2):143-51.e7.

17. Subha M, Arvind M. Role of Magnetic Resonance Imaging in Evaluation of Trigeminal Neuralgia with its Anatomical Correlation. Biomed Pharmacol J 2019;12:289-296.

18. Pandey M, Rao LP, Das SR, et al. Patterns of mandibular invasion in oral squamous cell carcinoma of the mandibular region. World J Surg Oncol 2007;5:12.

19. Patil SR, Maragathavalli G, Araki K, et al. Three-Rooted Mandibular First Molars in a Saudi Arabian Population: A CBCT Study. Pesqui Bras Odontopediatria Clin Integr 2018;18:4133.

20. Bombeccari GP, Candotto V, Giannì AB, et al. Accuracy of the Cone Beam Computed Tomography in the Detection of Bone Invasion in Patients with Oral Cancer: A Systematic Review. Euras J Med 2019;51:298.

21. Rohini S, Kumar VJ. Incidence of dental caries and pericoronitis associated with impacted mandibular third molar-A radiographic study. Res J Pharm Tech 2017;10:1081-1084.

22. Choudhury P, Panigrahi RG. Vanishing roots: first case report of idiopathic multiple cervico-apical external root resorption. J Clin Diagn Res 2015; 9(3): ZD17-ZD19.

23. Muthukrishnan A, Kumar LB, Ramalingam G. Medication-related osteonecrosis of the jaw: a dentist's nightmare. BMJ Case Rep. 2016; 2016: bcr2016214626.

24. Van Cann EM, Koole R, Oyen WJG, de Rooy JW, de Wilde JD, Slootwrg PJ, et al. Assessment of mandibular invasion of squamous cell carcinoma by various modes of imaging: construct- ing a diagnostic algorithm. Int J Oral Maxillofac Surg 2008 Jun;37(6):535-41.

25. Jin X, Liu D, Zhao X, Zhou Y, Jiang L, Jing L, et al. Analysis of clinicopathological characteristics associated with the outcome of oral squamous cell carcinoma and the establishment of tissue microarrays. Oncology Lett 2016 Nov; 12(5): 3175-3182.

26. Singh MP, Kumar V, Agarwal A, et al. Clinico-epidemiological study of oral squamous cell carcinoma: A tertiary care centre study in North India. J Oral Biol and Craniofac Res 2016;6:3235.

27. Johnson NW, Jayasekara P, Amarasinghe AAHK. Squamous cell carcinoma and precursor lesions of the oral cavity: epidemiology and aetiology. Periodontology 2000 2011;57:19-37.

28. Rao LP, Das SR, Mathews A, et al. Mandibular invasion in oral squamous cell carcinoma: investigation by clinical examination and orthopantomogram. Int J Oral Maxillofac Surg 2004;33:454-457.

29. Shah $\mathrm{P}$, Venkatesh $\mathrm{R}$, More $\mathrm{C}$, et al. A retrospective radiographic analysis of osseous changes in oral malignancy 2016;28(3): 236241.

30. Czerwonka L, Bissada E, Goldstein DP, Wood RE, Lam EW, $\mathrm{Yu}$ E, et al. High-resolution cone-beam computed tomography for assessment of bone invasion in oral cancer: Comparison with conventional computed tomography. Head Neck 2017 Oct;39(10):2016-2020.31.

31. Hakim SG, Wieker H, Trenkle T, et al. Imaging of mandible invasion by oral squamous cell carcinoma using computed tomography, cone-beam computed tomography and bone scintigraphy with SPECT. Clin Oral Investig 2014;18:961-967.

32. Joshi B. CBCT Analysis of Patients with Malignancy Involving Mandible: A Cross sectional descriptive study. Masters Dessertation, Tamilnadu Government Dental College and Hospital, Chennai 2018.

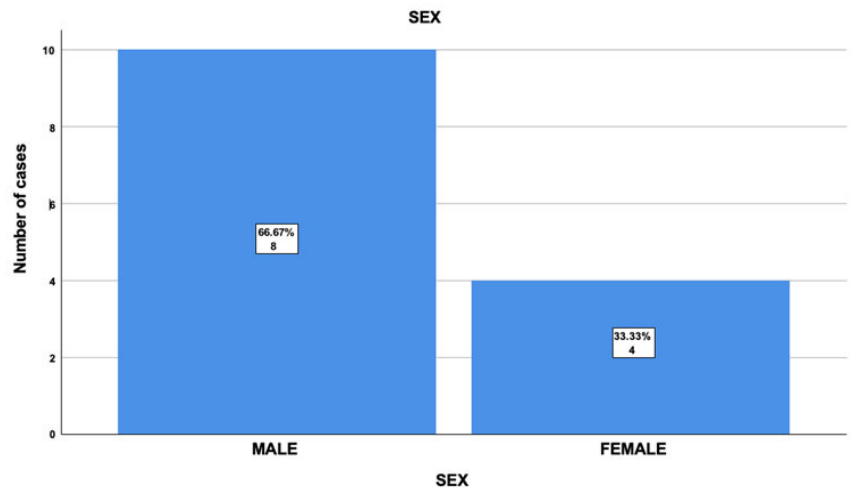

Graph 1: This graph depicts the frequency of sex distribution in the study population. X-axis depicts the gender of patients and Y-axis depicts the number of patients in the study population. Graph 1 shows that there were 8 male and 4 female participants in the study.

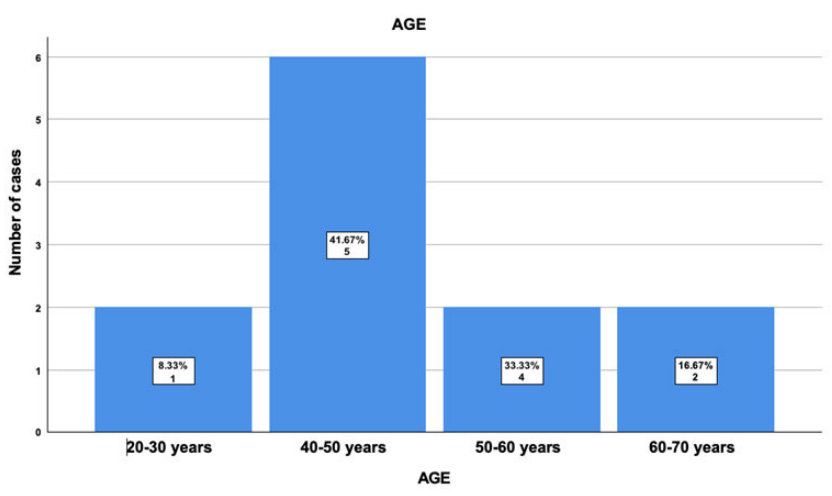

Graph 2: This graph shows the frequency of patients in different age groups with OSCC. X-axis depicts the age groups distributed among the study population and Y-axis depicts the number of cases. 5 patients were in the age group 40 - 50 years, 4 patients in 50-60 years, 2 patients in 60-70 years, and one patient in the $20-30$ years age group. 
3A:

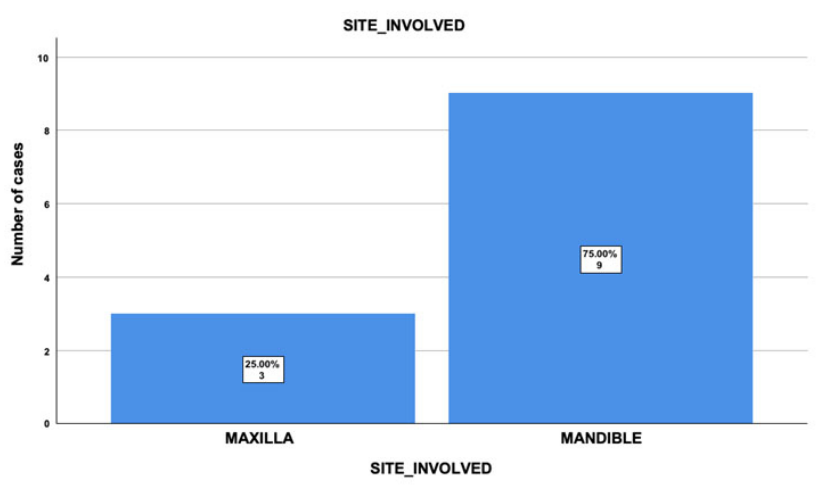

3B:

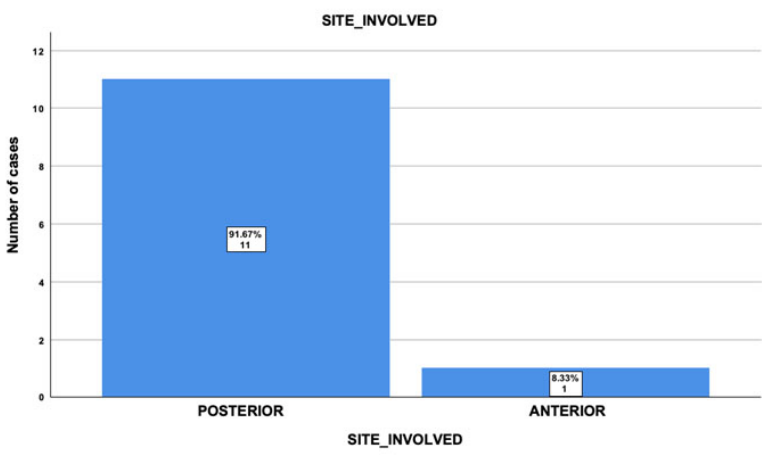

Graph 3: This graph depicts the involvement of sites in patients diagnosed with OSCC. X-axis shows the involvement of the site and $Y$-axis shows the numbers of cases in the study population. A, shows that in 9 patients the OSCC was affected in mandible followed by 3 patients in the maxilla. $B$, shows that 11 patients had posterior jaw involvement followed by anterior jaw involvement in one patient.

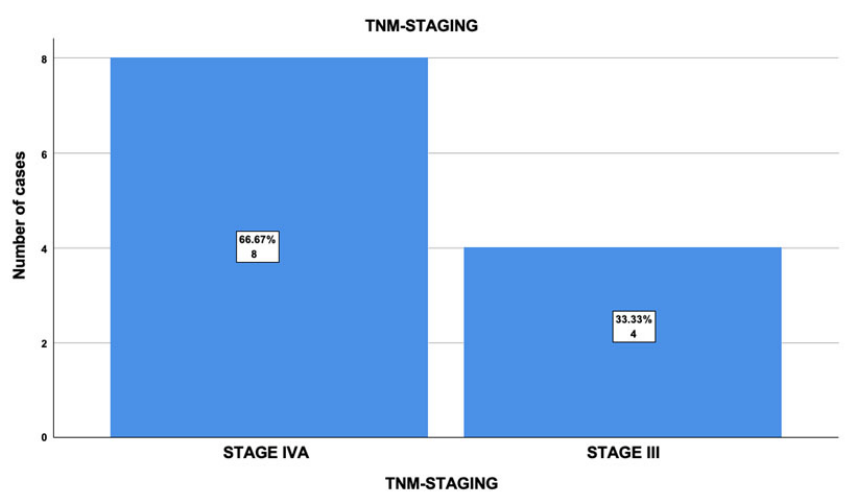

Graph 4: This graph depicts the TNM staging of OSCC patients. X-axis depicts the TNM staging and Y-axis depicts the numbers of cases in the study population. Graph 4 depicts that in 8 patients the carcinoma was in Stage IVA and for 4 patients in Stage III.

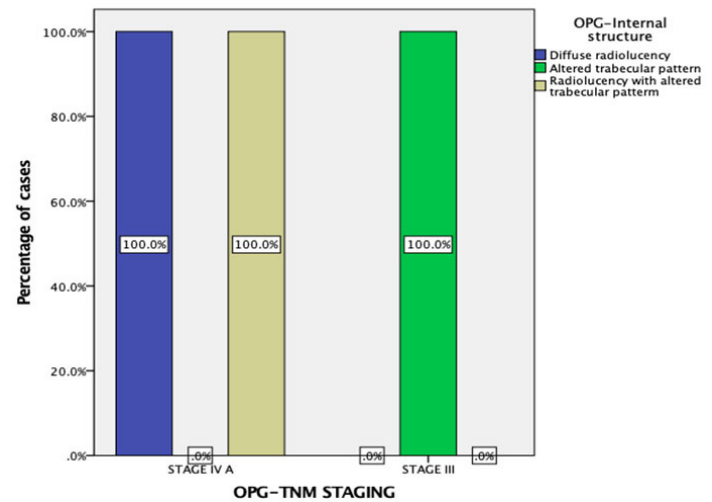

Graph 5: Bar graph depicting the association of OSCC and internal structure appearance in OPG.Blue denotes diffuse radiolucency; green depicts the altered trabecular pattern and brown depicts the presence of both altered trabecular pattern and radiolucency. X-axis represents the TNM staging and Yaxis represents the percentage of cases. Graph 5 shows that in Stage IV-A of OSCC there are both diffuse radiolucency seen with altered trabecular pattern (100\%) and in Stage-III there is a maximum of only altered trabecular pattern seen prominently $(100 \%)$. A Chi-square analysis to study the association between the internal structure of OSCC with TNM staging in OPG [chi square - 7.00; df-2; $p-0.030(p<0.05)$ ] is statistically significant. There is a positive correlation on comparing OSCC and internal structure appearance seen in OPG.

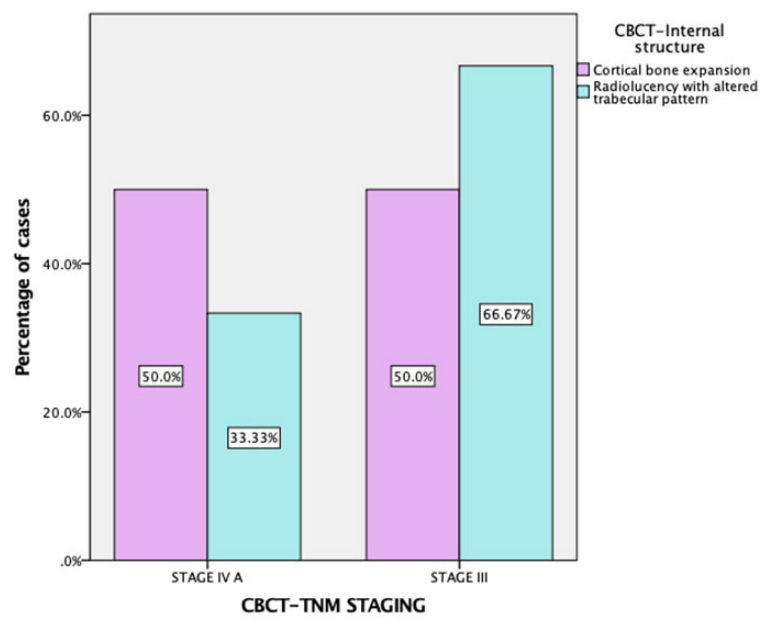

Graph 6: Bar graph depicting the association of OSCC and internal structure appearance in CBCT.Light Blue denotes the presence of radiolucency with altered trabecular pattern and purple depicts the presence of cortical bone expansion. $\mathrm{X}$-axis represents the TNM staging and $\mathrm{Y}$-axis represents the percentage of cases. Stage IVA and Stage III of OSCC there is $50 \%$ of involvement of cortical bone expansion seen and $33.33 \%$ of diffuse radiolucency seen with altered trabecular pattern in Stage IV-A and $66.67 \%$ in Stage-III. The Chi-square analysis [chi square - 0.139; df- $1 ; p-0.409(p>0.05)$ ] is statistically significant. There is a positive correlation on the comparison of OSCC and internal structure appearance seen in CBCT. 


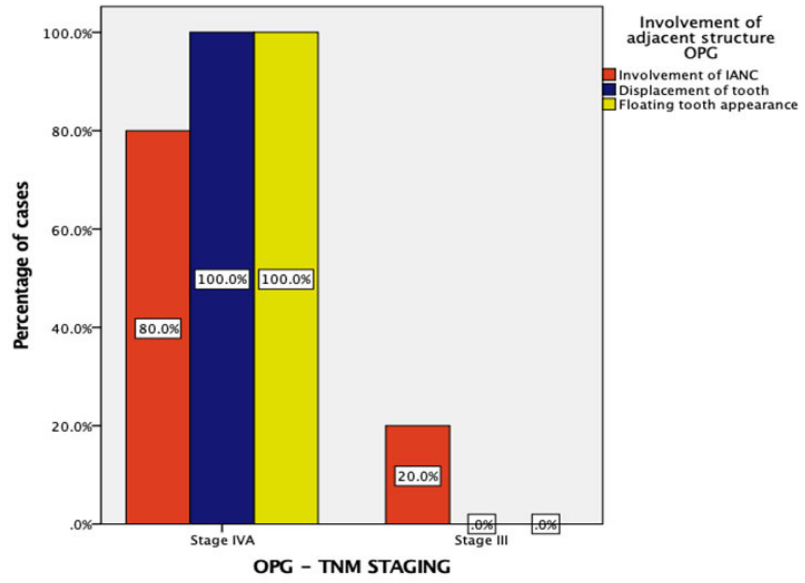

Graph 7: Bar graph depicting the association of OSCC and involvement of adjacent structures in OPG. Red denotes the involvement of the inferior alveolar canal (IANC) and dark blue depicts the displacement of tooth and yellow depicts the floating tooth appearance. X-axis represents the TNM staging and Y-axis represents the percentage of cases. Stage IVA there was the involvement of IANC (80\%), displacement of the tooth, and floating teeth appearance seen prominently (100\%). Stage III there was $20 \%$ involvement of IANC seen with no presence of displacement of teeth or floating teeth appearance. The chi-square analysis [chi-square-0.467; df-2; $p-.792(p>0.05)$ ] was statistically not significant. The involvement of adjacent structures was appreciated more in Stage IV-A than Stage-III of OSCC.

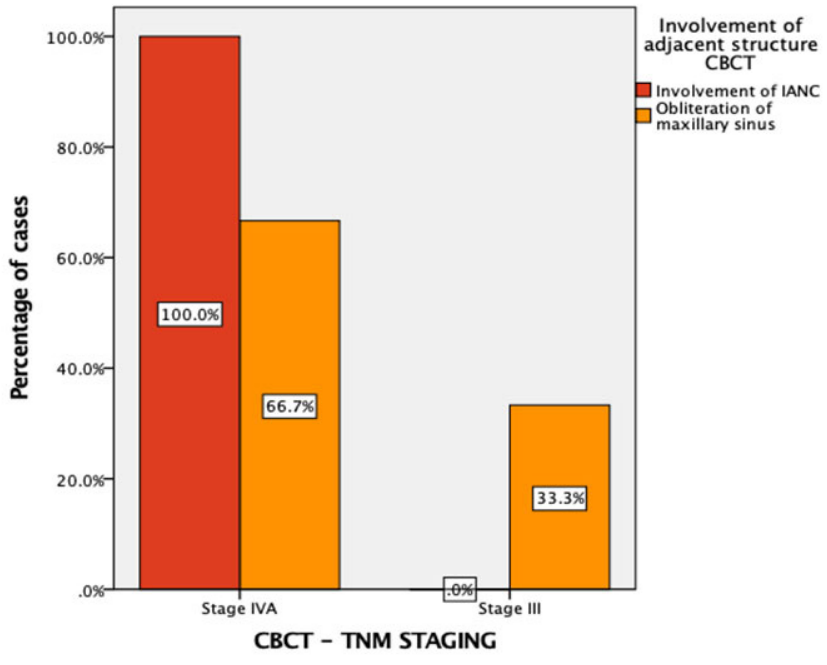

Graph 8: Bar graph depicting the association of OSCC and involvement of adjacent structures in CBCT. Red denotes the involvement of the inferior alveolar canal (IANC) and orange depicts the obliteration of the maxillary sinus floor. X-axis represents the TNM staging and $Y$-axis represents the percentage of cases. All cases in Stage IVA had the involvement of IANC and $66.7 \%$ of the obliteration of maxillary sinus floor. Stage III there was no involvement of IANC seen with $33.3 \%$ obliteration of the maxillary sinus floor. The chi-square analysis [chi-square-0.139; df-1; p-0.702 ( $p>0.05)$ ] was statistically not significant. CBCT imaging showed better involvement of the mandibular canal and obliteration of the maxillary sinus floor when compared to OPG in StagelVA of OSCC. 